\title{
Eberbard Kolb \\ Mächtepolitik und Kriegsrisiko am Vorabend des Krieges von 1870 Anstelle eines Nachworts
}

Wie die einzelnen Beiträge eindringlich vor Augen führen und wie die Diskussionen während des Kolloquiums selbst deutlich machten, waren es vor allem zwei Komponenten, die den internationalen Beziehungen im Europa der 1860er Jahre ihr signifikantes Gepräge gaben. Zum einen existierte eine ganze Reihe von Konfliktfeldern, und das angehäufte Konfliktpotential erzeugte immer wieder „kriegsträchtige Situationen" " . Zum anderen ist es jedoch bemerkenswert, daß es in jenen Jahren trotz der vielfältigen Spannungen und Interessengegensätze nicht zu einer kompakten „Blockbildung“ innerhalb des europäischen Staatensystems gekommen ist. Gewiß gab es einige ausgeprägte Grundtendenzen - die weitgehende Abstinenz der Flügelmächte Großbritannien und Rußland gegenüber den mitteleuropäischen Problemen wäre hier zu nennen sowie die seit 1867 bestehende französisch-preuBische Konfrontation -, aber charakteristisch für die Mächtebeziehungen in den 1860er Jahren war doch der rasche Wechsel der Konstellationen. Dadurch eröffneten sich Handlungsspielräume, die die politischen Akteure nutzen konnten - sei es zur Konfliktverschärfung, sei es zur Konfliktdämpfung.

Ausgehend von diesem Befund erscheint es sinnvoll, in Anknüpfung an die Beiträge, aber ohne sie nochmals akzentuierend zusammenzufassen oder den Diskussionsverlauf im einzelnen nachzuzeichnen, hier auf einige Aspekte kurz einzugehen, die für eine Re-Interpretation der Mächtebeziehungen und Mächtekonflikte in den 1860er Jahren von besonderer Bedeutung sind.

Wie nah war in den Jahren vor 1870 ein europäischer Krieg, zumal ein Krieg zwischen dem bonapartistischen Frankreich und der deutschen Führungsmacht Preußen? War dieser Krieg „unausweichlich“, oder konnte er durchaus vermieden werden, wenn und solange keiner der Kontrahenten ihn wirklich auslöste? Die Auffassungen der Historiker gehen in dieser Frage ebenso auseinander wie seinerzeit - bis in die Tage der Julikrise 1870 hinein - die Meinungen, Erwartungen,

${ }^{1}$ Dieser Begriff (in Analogiebildung zu „revolutionäre Situationen“) bei Dieter Ruloff, Wie Kriege beginnen (München 1985) 10. 
Hoffnungen der Zeitgenossen. Die Quellen bieten in der Tat ein höchst widersprüchliches Bild. Es mangelt nicht an Zeugnissen, in der Publizistik wie in der diplomatischen Korrespondenz jener Jahre, in denen die Überzeugung zum Ausdruck kommt, ein kriegerischer Konflikt zwischen Frankreich und dem Norddeutschen Bund werde sich nicht mehr lange vermeiden lassen. Aber diesen Zeugnissen stehen in großer Zahl solche gegenüber, die nicht eine derartige fatalistische Prognose präsentieren. So sind beispielsweise die Bekundungen der Abgeordneten des Norddeutschen Reichstags jüngst auf den Nenner gebracht worden, sie vermittelten den „subjektiven zeitgenössischen Eindruck“ eines Schwankens „zwischen latenter Kriegsfurcht bzw. -hoffnung und der Vermeidung der Gefahr eines unmittelbar bevorstehenden europäischen Zusammenstoßes“2. Mit dieser Formulierung dürfte die zeitgenössische Stimmungslage nicht nur diesseits, sondern auch jenseits des Rheins und im übrigen Europa recht zutreffend gekennzeichnet sein.

Hinzu tritt jedoch ein weiterer Sachverhalt, der im allgemeinen zu wenig beachtet wird. Wenn in den diplomatischen Akten der 1860er Jahre auch auf Schritt und Tritt vom Krieg als Möglichkeit, als leicht verfügbarem Instrument, als letztem Ausweg aus einer akuten Krisensituation die Rede ist, so besteht doch eine unübersehbare Diskrepanz zwischen der ständigen Evokation des Krieges und dem weitgehenden Verzicht auf konkrete Überlegungen, wie denn der entscheidende Schritt von der Krise zum Krieg getan werden konnte. Das ist erstaunlich. Im Zeitalter eines sich herausbildenden politischen Massenmarktes ließ sich der Bruch des Friedens nämlich keineswegs so einfach bewerkstelligen, wie anscheinend viele Politiker glaubten (wenn sie es tatsächlich glaubten) und wie manche Historiker annehmen. Bei der faktischen Auslösung eines allgemeinen europäischen Krieges oder eines Krieges zwischen zwei der großen europäischen Nationen war eine hohe Barriere zu überwinden. Dazu bedurfte es nicht nur des entschiedenen Kriegswillens zumindest einer der im Konflikt befindlichen Parteien, sondern der Bruch des Friedens mußte auch in einer stichhaltigen Weise begründet werden können, die sowohl das eigene Volk als auch die übrigen europäischen Nationen zu überzeugen vermochte; durch bloße Manipulation der öffentlichen Meinung war dies nicht zu bewirken. Trotz der gängigen Beschwörung des Krieges als eines universalen und vor allem eines jederzeit und bequem verfügbaren politischen Instruments bestand deshalb auch in den Jahren der sich verschärfenden Konfrontation zwischen Frankreich und dem Norddeutschen Bund wohl doch eine reale Chance, den Frieden zu erhalten.

Der europäischen Konstellation am Vorabend des Krieges von 1870 wird somit eine finale Betrachtungsweise nicht gerecht, die von der Prämisse der schließlichen Unvermeidlichkeit eines kriegerischen Konflikts zwischen Frankreich, das sich in seiner Hegemoniestellung bedroht fühlte, und der im Aufstieg befindlichen deut-

${ }^{2}$ Klaws Erich Pollmann, Parlamentarismus im Norddeutschen Bund 1867-1870 (Düsseldorf 1985) 236. 
schen Führungsmacht Preußen ausgeht und unter diesem Gesichtspunkt die Quellen selektiert, die Fakten gruppiert und die Zusammenhänge konstruiert. Gegen die Angemessenheit einer solchen Betrachtungsweise spricht nicht zuletzt auch die Tatsache, daß man in den europäischen Kabinetten durchaus um den schlechten Gesundheitszustand Napoleons III. wußte. Angesichts der Struktur des bonapartistischen Systems ließ es sich aber überhaupt nicht absehen, wie sich bei einem Ausscheiden Napoleons III. aus seiner Führungsfunktion die inneren Verhältnisse in Frankreich entwickeln würden, und von dieser Entwicklung wiederum hing es ab, ob sich die Aussichten für die Erhaltung des Friedens in Europa günstiger oder ungünstiger gestalteten. Es war deshalb am Ende des Dezenniums ein situationsadäquates politisches Kalkül, auf Zeitgewinn zu setzen, weil eine allmähliche Entspannung im deutsch-französischen Verhältnis, wenn auch kurzfristig wenig wahrscheinlich, so doch mittelfristig nicht gānzlich außerhalb des Bereichs der Möglichkeiten lag.

Dieser Gesichtspunkt sollte auch bei der Erörterung eines weiteren Problemkomplexes im Auge behalten werden. Wie sich in den Diskussionen des Kolloquiums deutlich herausschälte, ist die Beurteilung der Bismarckschen Süddeutschlandpolitik nach 1866 von ausschlaggebender Bedeutung für die generelle Bewertung der französischen und preußischen „Vorwärts-Strategie“ zwischen 1866 und 1870 und mithin auch für die Sicht der Entstehungsursachen des Krieges von 1870. Die Süddeutschlandpolitik Bismarcks rückte deshalb nicht von ungefähr in den Mittelpunkt des engagierten wissenschaftlichen Diskurses.

Wie ist Bismarcks Politik gegenüber den süddeutschen Staaten in den Jahren 1866 bis $1870 \mathrm{zu}$ qualifizieren - als weitgehend gescheitert, als stagnierend oder als durchaus erfolgreich? Jede dieser Deutungen hat - wie Lothar Gall mit Recht hervorhebt - „weitreichende Konsequenzen für die Interpretation der Bismarckschen Gesamtpolitik und insbesondere für seinen und Preußens Anteil an der Entstehung des Krieges von $1870^{\prime \prime 3}$. Lange dominierte in der deutschen Historiographie die Deutung, trotz aller Bemühungen Bismarcks um rasche Fortschritte in der deutschen Frage habe die Entwicklung nach 1867 im Zeichen eines zunehmenden Stagnierens des Einigungsprozesses gestanden, und einige Autoren - etwa Josef Becker in verschiedenen Aufsätzen ${ }^{4}$ und in seinen Diskussionsbeiträgen beim Kolloquium - verfechten sogar die These, am Vorabend des Krieges von 1870 sei offenkundig gewesen, daß Bismarck mit seiner Süddeutschlandpolitik weitgehend gescheitert war. Demgegenüber gelangt Lothar Gall, unter Berücksichtigung auch der Forschungsergebnisse von Rolf Wilhelm ${ }^{5}$ und Klaus Erich Pollmann ${ }^{6}$, zu einer

\footnotetext{
${ }^{3}$ Siehe oben S. 24.

4 Insbesondere Josef Becker, Zum Problem der Bismarckschen Politik in der spanischen Thronfrage 1870, in: HZ 212 (1971) 529-607.

5 Rolf Wilhelm, Das Verhältnis der süddeutschen Staaten zum Norddeutschen Bund (1867-1870) (Husum 1978).

${ }^{6}$ Siehe Anm. 2.
} 
wesentlich anderen Akzentuierung der Ziele und Ergebnisse von Bismarcks Süddeutschlandpolitik zwischen 1866 und 1870. Er arbeitet heraus, daß in der Einigungsfrage - entgegen landläufiger Ansicht - zwischen Bismarck und den Nationalliberalen keineswegs eine „Partnerschaft mit Divergenzen“" bestanden hat, sondern daß der Lagebeurteilung und der politischen Strategie beider Seiten höchst unterschiedliche, ja gegensätzliche Konzeptionen einer "Lösung" der deutschen Frage zugrunde lagen.

Bismarcks Süddeutschlandpolitik - so Gall - stand „eindeutig unter dem Primat machtpolitischer Strukturentscheidungen im Innern, wie er sie nach $1866 \mathrm{im} \mathrm{Be}-$ reich des Norddeutschen Bundes durchgesetzt hatte ${ }^{47}$. In der Konsequenz dieser Entscheidungen beschrānkte die Verfassung des Norddeutschen Bundes die Kompetenzen des Parlaments auf das Budgetrecht und die Mitwirkung bei der Gesetzgebung für Industrie, Handel, Gewerbe, Sozialpolitik und Recht; hingegen blieben die Außen- und Militärpolitik sowie die Besetzung der Regierungsämter eine Domāne der monarchischen Gewalt. Diese Machtverteilung zwischen Parlament, Regierung und Krone stand für Bismarck im Fortgang des Einigungsprozesses nicht mehr zur Disposition und war insofern kein „Provisorium“, das möglichst rasch und womöglich um nahezu jeden Preis - überwunden werden mußte. Deshalb strebte Bismarck in der Süddeutschlandfrage keine kurzfristigen Lösungen an. Er betrieb seine Süddeutschlandpolitik nicht in Gestalt eines Werbens um die Bevölkerung, die Parteien, die parlamentarischen Körperschaften der süddeutschen Staaten, sondern er suchte die süddeutschen Monarchen und ihre Ratgeber zu gewinnen, indem er deutlich machte, daß ein kleindeutscher Staat unter preußischer Führung, was die inneren Machtverhältnisse anbetraf, „kein Instrument der Veränderung, sondern eines der Bewahrung sein werde ${ }^{\text {c8 }}$. Gleichzeitig vertraute er auf die Wirkungen der dem Norden und Süden gemeinsamen Strukturelemente, insbesondere der institutionellen Klammer des Zollvereins.

Interpretiert man Zielsetzung und Methode der Bismarckschen Süddeutschlandpolitik in dieser Weise, dann läßt sich feststellen, daß auf dem eingeschlagenen Weg bis 1870 bemerkenswerte Erfolge erzielt wurden; dies hat Rolf Wilhelm schlüssig bewiesen. Im Horizont seiner eigenen Zielvorstellungen und Zeitperspektiven hatte Bismarck deshalb keinen Anlaß, im Jahr 1870 seine Süddeutschlandpolitik als gescheitert anzusehen oder ein so gefährliches Stagnieren zu diagnostizieren, daß es für ihn ein verführerischer Gedanke sein mußte, den Teufelskreis mit einer Flucht nach vorn zu durchbrechen.

Anders lagen die Dinge für die Nationalliberalen. Sie setzten gerade darauf, daß die Herstellung einer staatlichen Einheit zwischen dem Norddeutschen Bund und den süddeutschen Staaten die Möglichkeit eröffnen würde, den von ihnen 1866/67 eingegangenen Kompromiß bei der Fixierung der Machtverteilung zwischen Parla-

\footnotetext{
${ }^{7}$ Siehe oben S. 27.

8 Ebd.
} 
ment und Regierung zu revidieren, und zwar in Richtung einer stärkeren Beschneidung der monarchischen Gewalt. Bei den Nationalliberalen verband sich daher der Wille zu möglichst rascher Verwirklichung der nationalen Ziele mit der Erwartung, daß sie im Zuge der Errichtung eines kleindeutschen Staates auch manche ihrer verfassungspolitischen Absichten würden realisieren können. Aus diesem Grunde waren sie bereit, hohe außenpolitische Risiken einzugehen, sie drängten auf einen Konfrontationskurs gegenüber Frankreich und hofften auf eine Mobilisierung aller Kräfte durch ein massenbewegendes außenpolitisches Ereignis. Gerade an einer solchen Mobilisierung hatte Bismarck aus innenpolitischen Gründen kein Interesse, er erstrebte eine - notfalls langfristige - Lösung auf der Linie der für ihn vorrangigen innen- und machtpolitischen Strukturentscheidungen. Infolgedessen fühlte sich Bismarck in der Deutschlandpolitik nicht unter massivem Zeitdruck.

Die von Gall gebotene Interpretation der deutschlandpolitischen Zielsetzungen und Strategien Bismarcks einerseits, der Nationalliberalen andererseits besitzt nach meiner Auffassung einen außerordentlich hohen Grad von Plausibilität. Aus dieser Deutung ergeben sich wichtige Schlußfolgerungen für die Einschätzung der europäischen Situation am Vorabend des Krieges von 1870. Wenn erwiesen ist, daß Zielvorstellungen, Strategien und Zeitplanungen Bismarcks in bezug auf Süddeutschland keineswegs im wesentlichen identisch waren mit denjenigen der $\mathrm{Na}$ tionalliberalen, dann erscheint es völlig unzulässig, bei der Bewertung von Stoßrichtung und Perspektiven der Bismarckschen Süddeutschlandpolitik mit dem Maßstab der nationalliberalen Wünsche und Erwartungen zu operieren. Aus dem Drängen der Nationalliberalen nach entschiedeneren deutschlandpolitischen Aktivitäten sowie aus ihrer Enttäuschung über das Ausbleiben spektakulärer Erfolge und nach außen hin deutlich sichtbarer Fortschritte in der Deutschlandpolitik nach 1867 darf man somit keine Rückschlüsse ziehen auf Lagebeurteilung, Absichten und Motivation Bismarcks bei seinem außenpolitischen Agieren im Vorfeld des Krieges von 1870. Dies gilt auch und vor allem für die Untersuchung der Gründe, die Bismarck veranlaßt haben, seit Frühjahr 1870 die spanische Thronkandidatur des Hohenzollernprinzen zu fördern. Wollte Bismarck, indem er sich zur Förderung der von spanischer Seite ins Spiel gebrachten Hohenzollernkandidatur entschloß, die französische Regierung zum Krieg "provozieren“, weil er infolge des angeblichen Scheiterns seiner Süddeutschlandpolitik zunehmend unter einem Zwang zur Flucht nach vorn stand oder zumindest nach seiner subjektiven Lageeinschätzung glaubte, jetzt unter bewußter Inkaufnahme eines hohen Kriegsrisikos zur Offensive gegen Frankreich übergehen zu müssen? Eine solche Behauptung steht - zumal angesichts der eindringlichen Befunde von Gall, Wilhelm und Pollmann - auf schwachen Füßen. Sie bleibt eine bloße Mutmaßung, solange sie nicht durch beweiskräftige Quellenbelege in den Rang einer gesicherten Aussage erhoben werden kann. Derartige Quellenbelege sind bislang jedoch nicht beigebracht worden. Um Bismarcks Manövrieren in der spanischen Thronfrage zurei- 
chend zu interpretieren, bedarf es einer sorgfältigen Analyse anhand der Quellen im breiteren Kontext der europäischen Gesamtkonstellation des Jahres $1870^{9}$.

Die vorstehenden knappen Bemerkungen verfolgen lediglich den Zweck, an einigen Beispielen zu verdeutlichen, daß die in diesem Bande versammelten Beiträge einige wichtige Elemente für die Re-Interpretation der Mächtebeziehungen und Mächtekonflikte im Europa der 1860er Jahre präsentieren und deshalb in vielfältiger Weise dazu anregen können, den gesamten Problembereich neu zu durchdenken. Auch auf dem in jahrzehntelanger Forschung so intensiv durchgepflügten Feld der internationalen Beziehungen im 19. Jahrhundert lassen sich noch Entdekkungen machen, vor allem dann, wenn ein - gegenüber der diplomatiegeschichtlichen Arbeitsweise traditioneller Art - verfeinertes methodisches Instrumentarium eingesetzt wird.

Dazu gehört übrigens auch ein sorgfältig reflektierter Umgang mit jenen Begriffen, die bei der Interpretation von Zielvorstellungen und handlungsleitenden Motiven der politischen Akteure sowie bei der Charakterisierung von internationalen Konstellationen verwendet werden. Bezeichnet man beispielsweise die Förderung der Hohenzollernkandidatur durch Bismarck als eine Aktion, durch die Bismarck die französische Regierung zum Krieg ,provozieren“ wollte, so impliziert der Begriff der Provokation schon eine bestimmte Sicht der Zusammenhänge, obwohl eine derartige Feststellung bestenfalls im Ergebnis einer subtilen Analyse getroffen werden kann, nicht jedoch den Ausgangspunkt der Interpretation bilden darf. Ähnliches gilt für Begriffe wie „Defensive“ und „Offensive“, die gern, aber häufig recht unreflektiert gebraucht werden - ohne klare Präzisierung nämlich, ob hier der Historiker sein Urteil abgibt oder ob diese Begriffe auf die Ebene der Perzeption bezogen sind, auf die Einschätzung der Situation durch die Akteure selbst und die in der Konsequenz dieser subjektiven Lageeinschätzung gewählte politische Strategie. Gerade der Historiker der internationalen Beziehungen sollte sich immer bewußt sein, daß mit dem von ihm benutzten Begriffsvokabular - ob er es will oder nicht - bereits wesentliche Interpretationsvorgaben verbunden sind.

Der Ertrag eines Koiloquiums läßt sich gewiß nicht in wenigen Sätzen bündeln. Will man dennoch den Versuch machen, so etwas wie eine Summe zu ziehen, dann wäre wohl vor allem darauf abzuheben, daß bei der Untersuchung der zwischenstaatlichen Beziehungen und Konfliktlagen am Vorabend des Krieges von 1870 die Frage nach den Entwicklungsalternativen und der prinzipiellen Offenheit der Situation nicht außer acht gelassen werden darf und daß die Zielgerichtetheit

\footnotetext{
${ }^{9}$ Diese Analyse kann hier nicht geleistet werden. Wichtige neue Aufschlüsse zur spanischen Thronfrage bietet der Beitrag von Hans-Otto Kleinmann in diesem Band. Meine eigene Einschätzung von Bismarcks politischem Kalkül bei der Förderung der Hohenzollernkandidatur habe ich in meiner Studie „Der Kriegsausbruch 1870" (Göttingen 1970) ausführlich dargelegt und begründet. In allen wesentlichen Punkten halte ich - bei voller Würdigung der neueren Forschungs- und Diskussionsbeiträge zur Frage der Hohenzollernkandidatur - an der dort vorgelegten Interpretation fest.
} 
und Planmäßigkeit des außenpolitischen Verhaltens von Staaten und Regierungen - generell, aber gerade auch für diesen Zeitraum - nicht überschätzt werden sollte. Wie Graf Kielmansegg mit vollem Recht betont (und wie die in diesem Band enthaltenen Beiträge zur Genüge belegen), war das internationale System jener Jahre kein stark polarisiertes System, „es war nicht geprägt durch einen dominierenden Konflikt, der den Frontverlauf bestimmte und alle anderen Konflikte gleichsam in sich eingliederte. Probleme und Interessen waren eher diffus gelagert. Eine solche Konstellation erleichtert die Eingrenzung von Konflikten. Sie relativiert die Bedeutung eines jeden einzelnen Konfliktes." ${ }^{\text {(10 }}$

Diese Feststellung gilt nach meiner Überzeugung auch für den Konflikt, der sich nach 1866 zwischen dem bonapartistischen Frankreich und der deutschen Führungsmacht Preußen entwickelte, der sich aber vor dem Juli 1870 nicht zu einer permanent „kriegsträchtigen Situation“ zuspitzte. Angesichts der spezifischen Ausformung des internationalen Systems in den 1860er Jahren ist es deshalb durchaus vorstellbar, daß sich Veränderungen im Mächteverhältnis und selbst in der Rangordnung der Mächte nach 1866 in einem allmählichen Prozeß vollziehen konnten. Zwar hing über der europäischen Szene der späten 1860er Jahre das Damoklesschwert des Krieges, zumal eines nationalen Krieges zwischen Frankreich und den deutschen Staaten. Aber unausweichlich war dieser Krieg zum damaligen Zeitpunkt nicht.

${ }^{10}$ Siehe oben S. 121. 
